\title{
Cobalt nanoparticles deposited and embedded in AIN: Magnetic, magneto-optical, and morphological properties
}

\author{
Y. Huttel \\ Instituto de Microelectrónica de Madrid (Centro Nacional de Microelectrónica, Consejo Superior \\ de Investigaciones Científicas), Isaac Newton 8-PTM-28760 Tres Cantos, Madrid, Spain \\ Instituto de Ciencia de Materials de Madrid (Consejo Superior de Investigaciones Científicas), \\ 28049 Cantoblanco, Madrid, Spain \\ H. Gómez, C. Clavero, A. Cebollada, and G. Armelles \\ Instituto de Microelectrónica de Madrid (Centro Nacional de Microelectrónica, Consejo Superior \\ de Investigaciones Científicas), Isaac Newton 8-PTM-28760 Tres Cantos, Madrid, Spain \\ E. Navarro \\ Sección Departamental de Física Aplicada, Facultad de Veterinaria, U.C.M. Avenida de Puerta \\ de Hierro s/n, 28040, Madrid, Spain
}

M. Ciria, L. Benito, and J. I. Arnaudas

Departamento de Magnetismo de Sólidos, Departamento de Física de la Materia Condensada-ICMA, Univ. de Zaragoza-CSIC, 50071 Zaragoza, Spain

A. J. Kellock

IBM Almaden Research Center, San Jose, California

(Received 14 January 2004; accepted 11 May 2004)

\begin{abstract}
We present a structural, morphological, magnetic, and magneto-optical study of cobalt nanoparticles deposited on $50 \AA$ AlN/c-sapphire substrates and embedded in an AlN matrix. The dependence of the properties of Co nanoclusters deposited on AlN with growth temperature and amount of deposited Co are studied and discussed. Also we directly compare the properties of as grown and AlN embedded Co nanoclusters and show that the AlN matrix has a strong impact on their magnetic and magneto-optical properties. (C) 2004 American Institute of Physics. [DOI: 10.1063/1.1767975]
\end{abstract}

\section{INTRODUCTION}

Small magnetic particles (clusters, nanostructures, aggregates) are attracting considerable interest from both fundamental $^{1}$ and applied ${ }^{2}$ points of view. It is now well established that both the size as well as the symmetry reduction of magnetic and nonmagnetic materials can result in a drastic change in their physical properties. The modified magnetic properties of the magnetic materials ${ }^{3-5}$ are expected to open other technological possibilities, in particular in high-density data storage devices and magneto-optical sensors. Also, in order to achieve challenging technological goals, the magnetic particles need to be supported or embedded in other materials such as semiconductors or insulators. Whereas the first studies were performed mainly on free and supported magnetic clusters, increasing interest is now focusing on the magnetic properties of nanoclusters embedded in matrices. It is now well established that in free clusters, the three-dimensional metals present an enhanced magnetic moment per atom ${ }^{6,7}$ and that the magnetic orbital moment to magnetic spin moment ratio is highly enhanced. ${ }^{8}$ Co clusters display a nonmonotonic decrease of the average magnetization that has been related to changes in the crystallographic structure of the clusters. ${ }^{9,10}$ Several studies have been also performed on Co clusters deposited on different surfaces such as $\mathrm{Si}_{3} \mathrm{~N}_{4},{ }^{11} \mathrm{Au}(111),{ }^{12} \mathrm{Pd}(111),{ }^{13}$ or $\mathrm{Cu}(001) .{ }^{14}$ It is interesting to point out that for some systems, the influence of the substrate on the magnetic properties of the Co clusters has been evidenced through a strong hybridization with the
$\operatorname{Pd}(111)$ (Ref. 13) substrate or the reduction of the magnetic moment due to the increase of the number of neighbors around the magnetic Co atoms. ${ }^{14}$ In contrast to the magnetic properties of free and supported magnetic clusters, the magnetic properties of clusters embedded in different matrices are not well known. This is particularly true for the case of insulator matrices. Several studies have been performed on Co clusters embedded in metallic matrices such as $\mathrm{Cu},{ }^{15-18}$ $\mathrm{Ag},{ }^{19}$ and $\mathrm{Nb},{ }^{20}$ and also in a superconducting matrix ${ }^{21}$ whereas few studies have been performed on insulating matrices. ${ }^{18,22-25}$ These studies performed on Co clusters embedded in different matrices have highlighted the importance of the nature of the matrix on the resulting magnetic properties. While some matrices like $\mathrm{TiO}_{2}$ (Ref. 23) do not modify significantly the properties of the embedded Co clusters with respect to free clusters of the same dimensions, it has been shown that other matrices could modify the magnetocrystalline anisotropy ${ }^{16,21,22}$ or the magnetization. ${ }^{17,18}$ Some of these studies have pointed out the importance of the interface between the Co clusters and the matrix on the resulting magnetic properties. ${ }^{21,22,24-26}$ Moreover, the matrix also plays a very important role in the magneto-optical properties of the system: they can be tuned by changing the optical properties of the matrix. ${ }^{27}$ In that sense, the use of matrices with high refractive index, such as AlN, are expected to increase the magneto-optical response of the nanoparticles system.

In the present paper we explore and correlate the structural and morphological properties with the magnetic and 
magneto-optical properties of cobalt nanoparticles deposited on AlN/sapphire substrates and also embedded in an AlN matrix. We show that the growth conditions of the Co nanoparticles can modify the properties of the resulting systems; in particular, the size, the magnetic, and magneto-optical properties of the nanoparticles can be monitored by tuning the growth conditions during the sputtering process. Also the comparative study of the properties of the systems $\mathrm{Co} / \mathrm{AIN} /$ sapphire (nonembedded Co nanoparticles) and AlN/Co/AlN/sapphire (AlN embedded Co nanoparticles) allows us to clearly identify the impact of the AlN matrix on the Co nanoparticles properties.

The paper is organized as follows: Sec. II describes the experimental details; Sec. III is devoted to the effect of the growth temperature of the Co nanoparticles on their properties; in Sec. IV we show the evolution of the properties of the nanoparticles versus the amount of Co; Sec. V is centered on the effect of the AlN matrix on the magnetic and magnetooptical properties of the Co particles; and the conclusions are presented in the last section.

\section{EXPERIMENTAL DETAILS}

All sample preparations were performed by triode sputtering in an ultrahigh vacuum chamber with a base pressure in the low $10^{-9}$ mbar. The cobalt was deposited on sapphire(0001) substrates $(0.5 \mathrm{~nm}$ thick, Marketech International) previously covered by a $50 \AA$ thick AlN buffer layer. The AlN was grown by reactive sputtering using an $\mathrm{Al}$ target and a $\left(\mathrm{Ar}+\mathrm{N}_{2}\right)$ plasma; the growth parameters (temperatures, composition of gases, deposition rates) were chosen in order to optimize the epitaxial growth of AlN as described elsewhere. ${ }^{28}$ In this study all the AlN layers were deposited keeping the substrate temperature at $400{ }^{\circ} \mathrm{C}$. The cobalt was deposited at a deposition rate of $22 \AA \mathrm{min}^{-1}$ and in some cases a protective $50 \AA$ AlN layer was deposited on top of cobalt and in the same conditions as the buffer layer. The structural and morphological characterizations were performed ex situ by x-ray diffraction (XRD) and atomic force microscopy (AFM). The XRD characterization was performed by using $\mathrm{Cu} K \alpha$ radiation $(\lambda=1.5418 \AA)$, BraggBrentano configuration with a secondary $\mathrm{Cu}$ monochromator and $1 / 4^{\circ}$ slits. The AFM surface micrographs were taken using a commercial instrument (Nanoscope III; Digital Instruments, Santa Barbara, CA). Single-beam silicon nitride cantilevers (Olympus) with a spring constant $0.1 \mathrm{Nm}^{-1}$ were used to perform the experiments in the contact mode operation. The magneto-optical characterizations were performed ex situ and at room temperature (RT) in the polar and transverse Kerr configurations. Superconducting quantum interference device (SQUID) and Rutherford backscattering spectroscopy (RBS) experiments were also performed. Although no SQUID nor RBS experimental results are shown in this contribution, the experiments were performed on specific samples in order to check the presence of an exchange bias and to precisely determine the amount of deposited Co. Magnetic force microscopy (MFM) images were acquired, at room temperature, in air, and with zero applied magnetic field, by using a Nanotec Electronica, S. L. commercial scan-

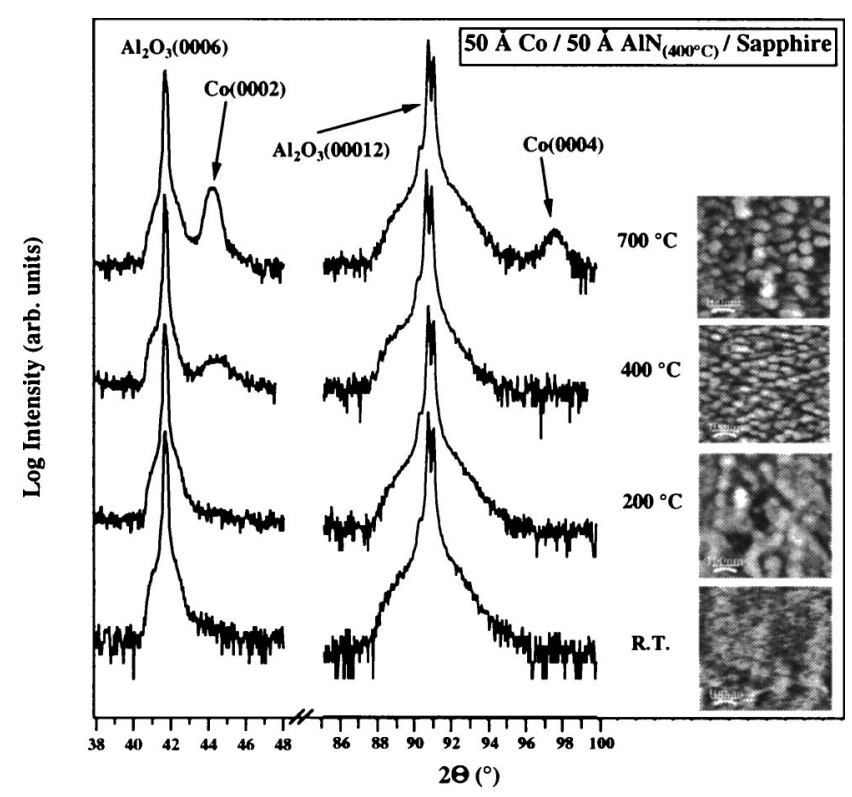

FIG. 1. High angle XRD patterns of $50 \AA \mathrm{Co} / 50 \AA \mathrm{AIN}_{400}{ }^{\circ} \mathrm{C} / \mathrm{sapphire}$ for cobalt deposited at different temperatures together with the corresponding two-dimensional atomic force microscopy images. Curves are vertically offset for clarity.

ning microscope equipped with a $12 \mu \mathrm{m}$ scanner. Tapping mode, etched silicon cantilevers (Nanosensors ${ }^{\mathrm{TM}}$ ) were used. The tips were coated with cobalt, deposited by magnetron sputtering, and magnetized before scanning the samples. The magnetic images were taken at a constant distance of $80 \mathrm{~nm}$ between the tip and the film level determined by a previous topographical scan.

\section{INFLUENCE OF THE Co DEPOSITION TEMPERATURE}

It is possible to obtain three-dimensional (3D) growth of metals on insulators by high temperature deposition. We have previously observed this effect, for example, for the growth of $\mathrm{Fe}$ on c-sapphire and $\mathrm{MgO}$ at elevated temperatures. ${ }^{29}$ A similar approach will be followed in this work where we will investigate the influence of the Co deposition temperature on the crystallinity and morphology.

Since the final goal is to obtain Co nanoparticles embedded in an AlN matrix, we first grew a $50 \AA$ thick and continuous AlN layer on a (0001) oriented sapphire substrate, on top of which Co nanoparticles could be grown and later covered with AlN. A $400{ }^{\circ} \mathrm{C}$ temperature was selected for the deposition of this first AlN layer as a compromise between flatness and crystallinity optimization: AlN deposition at $700{ }^{\circ} \mathrm{C}$ produces highly crystalline, but islanded AlN films, while deposition at $400{ }^{\circ} \mathrm{C}$ gives rise to flat and continuous films but with small grain sizes. ${ }^{28}$ Once this first AlN layer is grown, a series of $50 \AA$ thick Co films were grown at different temperatures (RT, 200, 400, and $700{ }^{\circ} \mathrm{C}$ ). In Fig. 1 we show the corresponding high-angle XRD longitudinal scans together with the associated two-dimensional AFM images. Besides the (0006) and (00012) substrate diffraction peaks, additional features are only present for the samples grown at 400 and $700{ }^{\circ} \mathrm{C}$. These features correspond, respectively, to the (0002) and (0004) hcp Co peaks and indicate the textured 
Co growth with the $c$ axis along the surface normal. The positions of these reflection peaks correspond exactly to those of bulk hcp Co, indicating the relaxed nature of the grown Co structures. In the $400{ }^{\circ} \mathrm{C}$ sample only the (0002) peak is observed, but in the $700{ }^{\circ} \mathrm{C}$ the (0004) diffraction peak is also visible due to the higher crystallinity of the Co grown at this temperature. Grain sizes estimated by applying Scherrer's equation to the (0002) breadth's reflections yield values of 60 and $85 \AA$ for 400 and $700{ }^{\circ} \mathrm{C}$, respectively. The evidence of grains having sizes larger than the nominal Co thickness is a first indirect indication of the 3D growth mode of $\mathrm{Co}$ at these temperatures; this implies uncovered substrate areas and therefore excess of material accumulated on top of the islands producing heights larger than the nominal thickness. The absence of Co diffraction peaks for samples grown at RT and $200{ }^{\circ} \mathrm{C}$ indicate small Co grain size for films deposited at low temperatures.

A much more direct evidence of the 2D or 3D growth mode for these structures comes with the use of a real space microscopic characterization technique. We have therefore investigated the morphology of the deposits using AFM that is the best-suited tool for this purpose. The results are also presented in Fig. 1. Whereas the sample grown at RT exhibits a flat surface, with a rms roughness of $5 \AA$, the morphology of the sample grown at $200^{\circ} \mathrm{C}$ evidences some marked features (rms roughness of $10 \AA$ ) producing a morphology that differs from a pure 2D structure. The change in morphology is even more evident for 400 and $700{ }^{\circ} \mathrm{C}$ deposition temperatures, where clear islands with typical lateral sizes of 36 and $47 \mathrm{~nm}$ for 400 and $700{ }^{\circ} \mathrm{C}$, respectively, and vertical sizes of 2 and $6 \mathrm{~nm}$ for 400 and $700{ }^{\circ} \mathrm{C}$, respectively, are observed.

Once the influence of the deposition temperature on the structure and morphology of the Co deposits was studied, we proceeded to study the influence on the magnetic and magneto-optical properties by means of ex situ polar Kerr measurements. In Fig. 2 we show the polar Kerr hysteresis loops measured at a fixed photon wavelength of $650 \mathrm{~nm}$ for the previously described samples. The first obvious consequence of the 2D-3D transition observed using $\mathrm{x}$-ray diffraction is the change in the shape of the hysteresis loops, the 2D films being far from saturation while the saturation field for the 3D structures is smaller, specially for the sample grown at $700{ }^{\circ} \mathrm{C}$, since demagnetizing fields are less important in islanded structures than in thin films. On the other hand, besides the evident change in the shape of the hysteresis loops, a gradual variation in the saturation Kerr signal is observed as a function of deposition temperature. Even though none of the samples is completely saturated at the highest achievable field $(1.5 \mathrm{~T})$, the $700{ }^{\circ} \mathrm{C}$ sample is almost saturated, and the individual approaches to saturation of the other samples clearly point to different saturation Kerr rotations for all of them. In the polar geometry, the Kerr rotation is in principle proportional to the sample magnetization, but it also strongly depends on the morphology. ${ }^{27}$ The dependence of the Kerr rotation with the morphology might be not negligible in our case due to the change from $2 \mathrm{D}$ to $3 \mathrm{D}$ entities and also regarding the size of the particles. While a deeper investigation of the variation of the polar Kerr re-

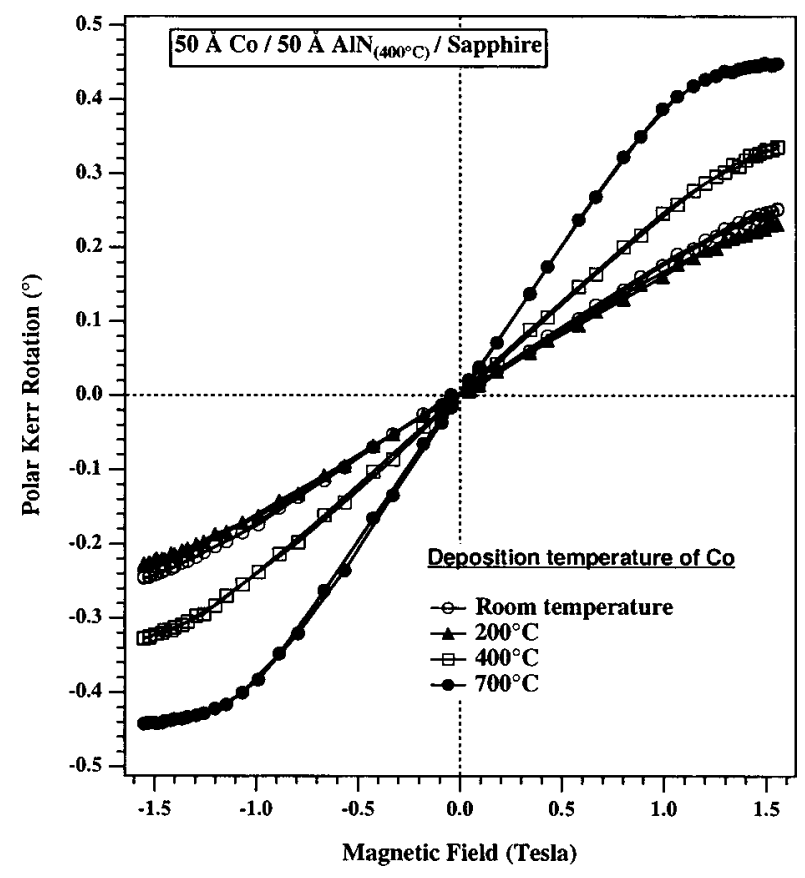

FIG. 2. Hysteresis loops measured in the polar Kerr configuration for $50 \AA \mathrm{Co} / 50 \AA \mathrm{AIN}_{400}{ }^{\circ} \mathrm{C} /$ sapphire for different Co deposition temperatures. The wavelength of light was $650 \mathrm{~nm}$.

sponse goes beyond the scope of our work, it is clear from the present results that the Kerr rotation of Co deposited on AlN depends drastically on the Co deposition temperature.

So far we have determined that high deposition temperature leads to enhanced crystallinity and Kerr rotation in this specific system. For further use of Co nanoparticles, for example, in magneto-optical (MO) sensors and in optoelectronic devices it is important to ensure the insertion of nanoparticles having the highest magneto-optical response. Therefore in the following we will focus our study on the properties of cobalt deposited at $700{ }^{\circ} \mathrm{C}$, i.e., of cobalt having a higher crystallinity and that self-organizes into well defined nanoparticles. We will investigate the modification of the properties of the deposits with the amount of deposited cobalt and also the influence of an embedding AlN matrix (or in our case of an AlN capping layer).

\section{INFLUENCE OF THE CO NOMINAL THICKNESS}

In the preceding section we have found that $700{ }^{\circ} \mathrm{C}$ is the best suitable temperature to obtain Co nanoparticles on AlN exhibiting the largest MO response for all the tested growth temperatures. The next step in this section will be to select this growth temperature and vary the Co nominal thickness, to control the Co particle size and determine the structure, morphology, and magnetic as well as magnetooptical properties as a function of the average particle size. In Fig. 3 we show the high-angle XRD longitudinal scans corresponding to different nominal Co thickness deposited with a substrate temperature kept at $700{ }^{\circ} \mathrm{C}$. Co is deposited on AlN buffer layers like for the previous series. Both (0002) and (0004) hcp Co diffraction peaks are observed for thicknesses down to $30 \AA$, becoming weak shoulders for 10 and $5 \AA$ thick films. As expected, the intensity and width of these 


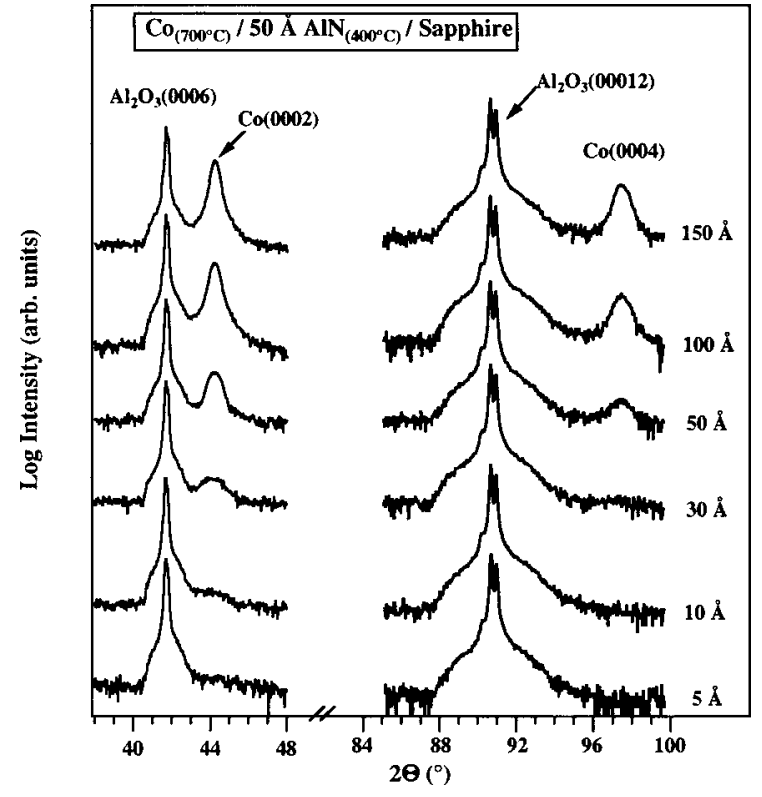

FIG. 3. High angle XRD patterns of $\mathrm{CO}_{700}{ }^{\circ} \mathrm{C} / 50 \AA \mathrm{AIN}_{400}{ }^{\circ} \mathrm{C} /$ sapphire for different nominal thickness of cobalt. Curves are vertically offset for clarity.

peaks evolve with cobalt coverage, being more intense and narrower for the thicker films. Besides, the peak positions depend on nominal thickness, appearing at higher scattering angles (smaller out of plane interplanar distances) for higher nominal thickness. The out of plane lattice parameter and the estimated grain size for the different thicknesses are given in Table I. No value was extracted for the 5 and $10 \AA$ thick layers due to the low peak intensity and strong overlapping with the substrate (0006) diffraction peak. Clearly, the perpendicular interplanar distances are expanded with respect to bulk value $(c=4.06 \AA)$, the largest expansion occurring for the thinner film. When increasing the thickness of the cobalt, the cobalt lattice parameter tends to recover its bulk value. Regarding the calculated grain size, the obtained values are larger than the nominal thickness for the 20,30 , and $50 \AA$ films, and smaller for the 100 and $150 \AA$ ones. This indicates that the thinner films are formed by single crystalline islands that are higher than the corresponding nominal thickness, while the two thicker layers probably consist of large islands formed by several single crystalline grains piled along the growth direction.

To get further information, a detailed characterization of the surface morphology at the nanometric level was performed using AFM. In Fig. 4 the AFM images corresponding to selected samples with nominal Co thickness of 5, 30, 50, and $100 \AA$ are presented. The AFM images reveal that, under

TABLE I. Perpendicular (out of plane) lattice parameter and mean grain size upon Co nominal thickness.

\begin{tabular}{ccc}
\hline \hline Co nominal thickness $(\AA)$ & Lattice constant $(\AA)$ & Mean grain size $(\AA)$ \\
\hline 20 & 4.120 & 43 \\
30 & 4.116 & 50 \\
50 & 4.080 & 86 \\
100 & 4.095 & 67 \\
150 & 4.093 & 81 \\
\hline
\end{tabular}

\section{$\mathrm{Co}_{700^{\circ} \mathrm{C}} / 50 \AA \mathrm{AlN}_{400^{\circ} \mathrm{C}} /$ Sapphire}
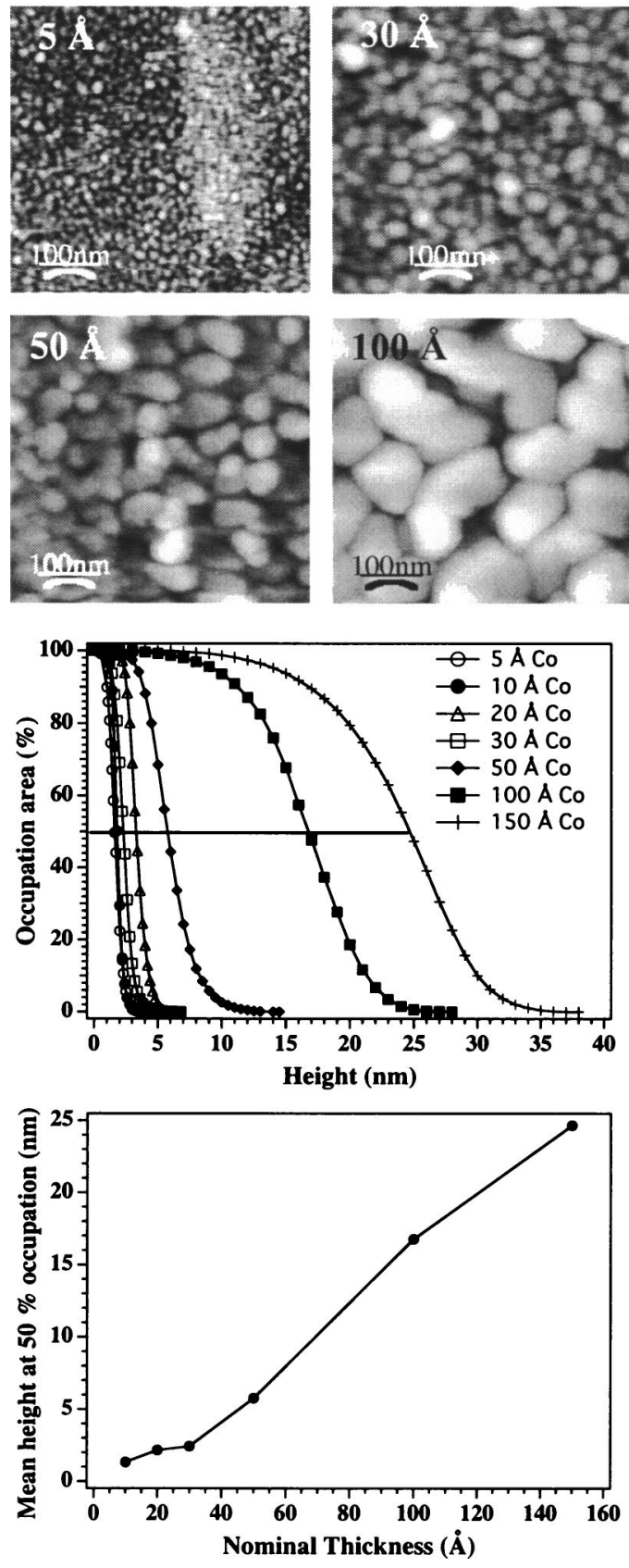

FIG. 4. Top: selected set of two-dimensional atomic force microscopy images corresponding to Co deposited on $\mathrm{AIN}_{400}{ }^{\circ} \mathrm{C} /$ sapphire and for different Co nominal thickness: 5, 30, 50, and $100 \AA$. The substrate temperature was kept at $700{ }^{\circ} \mathrm{C}$ during Co deposition. Center: occupation area vs height of Co nanoparticles. Bottom: mean height at $50 \%$ occupation vs Co nominal thickness.

the considered growth conditions, for low coverage the cobalt aggregates into small clusters that coalesce with increasing amounts of cobalt and form bigger clusters. The results of the analysis of the AFM images (see Table II) show that the Co nanoparticles have a mean diameter always bigger than the mean height. Also the occupation area can be derived from the AFM images measuring the area occupied by Co in slices performed at different heights. The results are illustrated in Fig. 4 (center). Assuming that the mean height 
TABLE II. Mean diameter and height of Co nanoparticles upon Co nominal thickness.

\begin{tabular}{rccc}
\hline \hline $\begin{array}{c}\text { Co nominal } \\
\text { thickness }(\AA)\end{array}$ & $\begin{array}{c}\text { Mean } \\
\text { diameter }(\mathrm{nm})\end{array}$ & $\begin{array}{c}\text { Mean height }(\mathrm{nm}) \\
\text { at 50\% occupation }\end{array}$ & $\begin{array}{c}\text { Mean diameter/ } \\
\text { Mean height }\end{array}$ \\
\hline 5 & 11 & 1.6 & 6.8 \\
10 & 23 & 1.3 & 17.6 \\
20 & 22 & 2.1 & 10.4 \\
30 & 30 & 2.4 & 12.5 \\
50 & 47 & 5.7 & 8.2 \\
100 & 100 & 16.7 & 5.9 \\
150 & 94 & 24.6 & 3.8 \\
\hline \hline
\end{tabular}

is the height found at $50 \%$ of occupation area, we have generated the graph that gives the mean height of the Co nanoparticles versus the nominal thickness of deposited Co (bottom of Fig. 4). We observe that the mean height is a monotonic function of the nominal thickness; this implies that the height of the deposited nanoparticles can be monitored by simply adjusting the deposition time of cobalt.

On the other hand, for the design of a system formed by nanoparticles embedded in a matrix, both particle height and diameter are relevant parameters because they determine the shape or aspect ratio of the particle. This aspect ratio, calculated as the diameter vs height ratio, is also shown in Table II. This ratio has a value around 7 for the thinnest Co layer (5 $\AA$ ), and abruptly increases to almost 18 for the $10 \AA$ case. This increment is exclusively due to a lateral increase of the islands size, since there is no appreciable change in the height, and implies a completion of the Co layer from 5 to $10 \AA$, adopting a more 2D character. Nevertheless, from this point on this trend is reversed and the islands grow faster in the vertical than in the horizontal dimension, with a growth mode closer to $3 \mathrm{D}$, yielding a gradual decrease of the diameter vs height ratio that reaches its minimum at $150 \AA$.

An open question at this point is the lateral contact between the Co nanoparticles, which cannot be fully clarified with AFM experiments due to the unavoidable convolution of the particles profile with the shape of the probing tip. We have tried to indirectly answer this question using MFM on selected samples. As an example, in Fig. 5 we show AFM [Fig 5(a)] and MFM [Figs. 5(b) and 5(c)] images of $50 \AA$ of Co grown at $700{ }^{\circ} \mathrm{C}$ on $50 \AA \mathrm{AlN} /$ sapphire. While the AFM image displays the expected well-defined Co nanoclusters, the corresponding MFM images show larger structures corresponding to magnetic domains with an in-plane magnetization. The significantly larger size of the domains with respect to that of the particles obtained from the AFM image clearly indicates that they are magnetically connected, probably through their bases. Since the sample is measured $e x$ situ and without any capping layer to protect the Co particles from oxidation, it is important to point out the relatively small exchange bias field ( -195 Oe) observed in transverse hysteresis loops measured in the same sample that was cooled in a $15 \mathrm{kOe}$ field from $350 \mathrm{~K}$ (above the antiferromagnetic transition temperature of $\mathrm{CoO}$ ) down to the measuring temperature of $5 \mathrm{~K}$. Such exchange bias is expected due to the presence of Co oxide resulting from the oxidation of the sample surface and is well known in cobalt oxides. ${ }^{30,31}$

\section{$50 \AA \mathrm{Co} / \mathrm{AlN} / \mathrm{Sapphire}$}

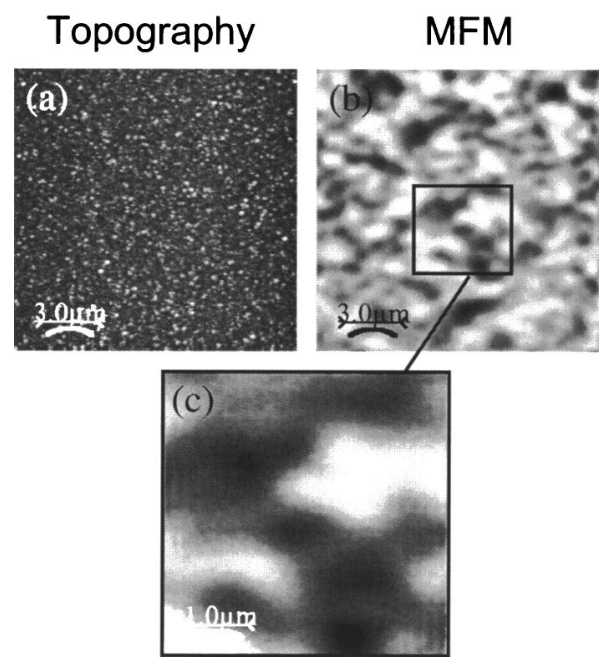

FIG. 5. (a) $\mathrm{AFM}$ and (b) and (c) $\mathrm{MFM}$ images of $50 \AA \mathrm{Co}_{700}{ }^{\circ} \mathrm{C} / 50 \AA$ $\mathrm{AIN}_{400}{ }^{\circ} \mathrm{C} /$ sapphire.

\section{INFLUENCE OF AN AIN CAPPING LAYER; Co NANOCLUSTERS EMBEDDED IN AN AIN MATRIX}

Up to now we have investigated the growth of Co nanoparticles on $50 \AA \mathrm{AlN} / \mathrm{sapphire}$ and found that it can be achieved by deposition at high temperature $\left(700{ }^{\circ} \mathrm{C}\right)$ and that the size of the clusters can be controlled with the deposition time or the deposited nominal thickness. We have characterized the nanoclusters from morphological, crystalline, and magnetic points of view. We will now study the same Co nanoclusters when embedded in an AIN matrix, obtained by depositing an AlN capping layer onto the Co particles grown on AlN. These kind of systems are specially attractive since their magneto-optical response can be tailored by adequate selection of both particle size and shape as well as refractive indices of the different materials. The samples studied in this section are Co nanoparticles of different sizes, grown at $700{ }^{\circ} \mathrm{C}$ on previously described AlN buffer layers, and capped with a $50 \AA$ AlN film grown at $400{ }^{\circ} \mathrm{C}$. The corresponding XRD scans are displayed in Fig. 6. No significant changes could be observed by comparing x-ray results for the AlN capped and uncapped Co clusters indicating that the AlN capping layer did not change significantly the crystallographic properties of the Co nanoparticles. In Fig. 7 we present the AFM and MFM images for a selected sample with $50 \AA$ nominal Co thickness. The morphology of the capped structure exactly replicates that of the bare Co particles, studied in the preceding section, and demonstrate the conformal character of the AlN capping layer; in fact, this behavior has been observed for all samples independently of the size of the nanoclusters. Following the same approach used in the preceding section, MFM images allow the determination of the physical contact of the particles, and in this case the observed magnetic domains are much bigger than the nanoclusters such as for the uncapped samples, indicating that the AlN capping layer does not change the formation of big magnetic domains.

We now turn to a more detailed study of the magnetic 


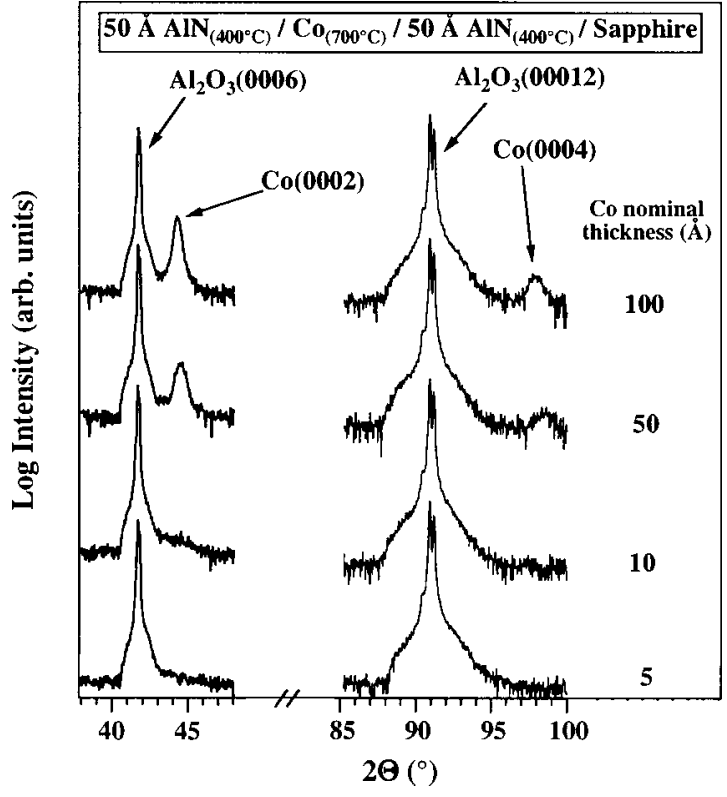

FIG. 6. High angle XRD patterns of $50 \AA \mathrm{AIN}_{400}{ }^{\circ} \mathrm{C} / \mathrm{Co}_{700}{ }^{\circ} \mathrm{C} / 50 \AA$ $\operatorname{AIN}_{400}{ }^{\circ} \mathrm{C} /$ sapphire for different nominal thickness of cobalt. Curves are vertically offset for clarity.

and magneto-optical properties of the AlN capped and uncapped Co nanoclusters. Figure 8 shows the hysteresis loops measured in transverse Kerr geometry and at a photon wavelength of $632.8 \mathrm{~nm}$. Figures 8(a) and 8(b) correspond to samples with Co nominal thickness of 5 and $10 \AA$, respectively. It is worth mentioning that the hysteresis loops measured in the transverse geometry did not display an in-plane magnetic anisotropy indicating therefore the lack of easy and hard magnetization axis even for the highest coverage studied in this work (150 $\AA$ ); this finding is consistent with the formation of randomly oriented and isotropic Co nanoclusters. The hysteresis loops displayed in Fig. 8 clearly show that the AlN capping layer of $50 \AA$ has a drastic effect on the magnetic properties of the Co nanoclusters. In the case of the smallest clusters [mean diameter of $11 \mathrm{~nm}$, Fig. 8(a)] the

\section{$50 \AA \mathrm{AlN} / 50 \AA \mathrm{Co} / \mathrm{AlN} / \mathrm{Sapphire}$}

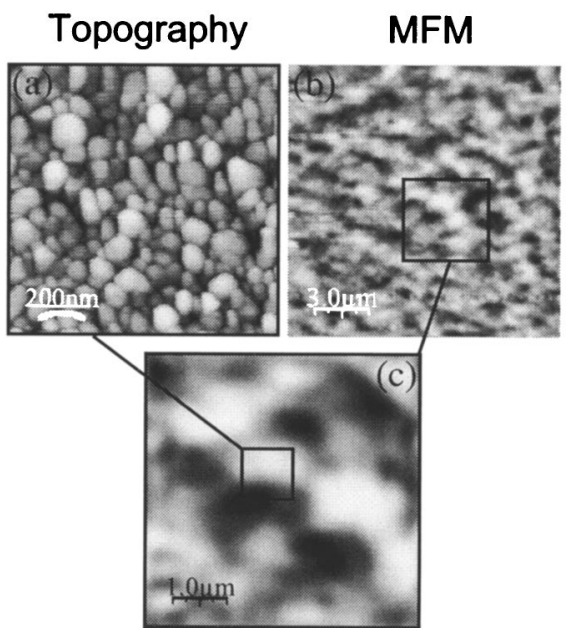

FIG. 7. (a) AFM and (b) and (c) MFM images of $50 \AA$ $\mathrm{AIN}_{400}{ }^{\circ} \mathrm{C} / 50 \AA \mathrm{Co}_{700}{ }^{\circ} \mathrm{C} / 50 \AA \mathrm{AIN}_{400}{ }^{\circ} \mathrm{C} /$ sapphire.

\section{Transversal Kerr}
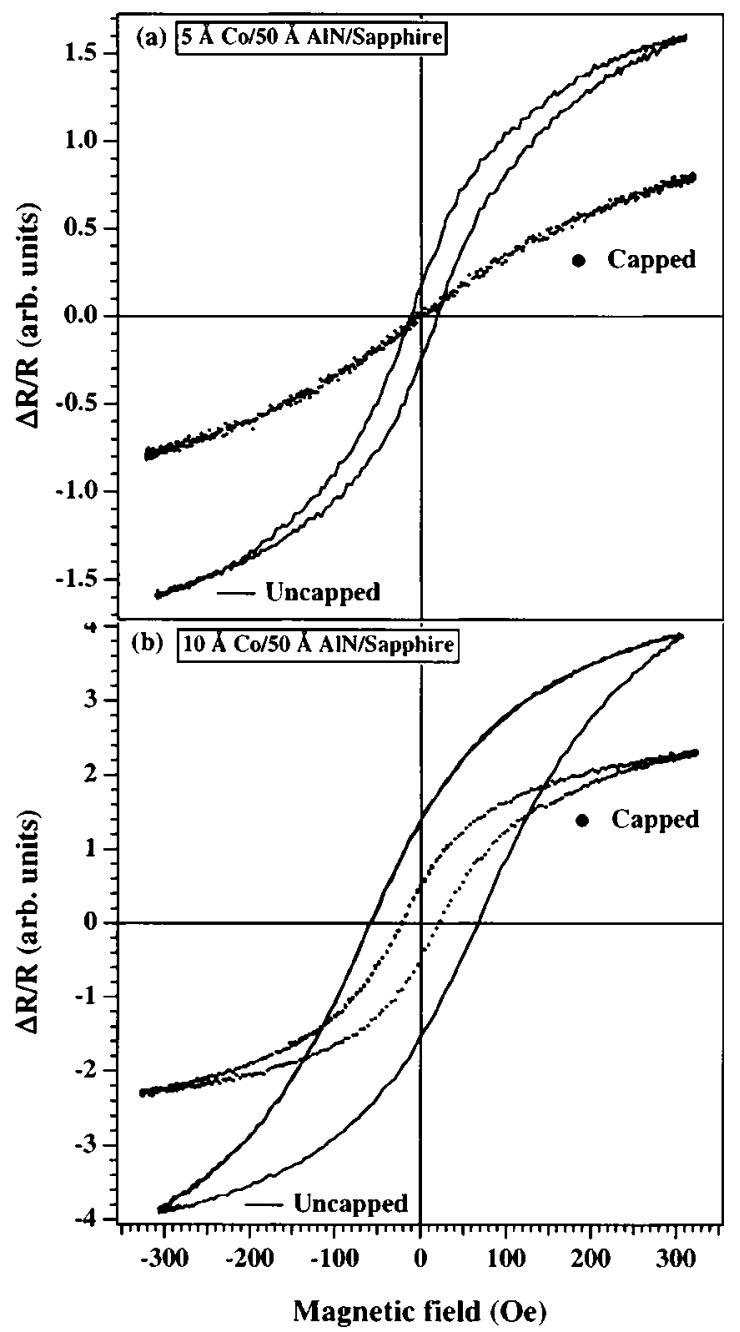

FIG. 8. Hysteresis loops measured in the transverse Kerr configuration for capped and uncapped $\mathrm{Co} / 50 \AA$ AlN/sapphire samples with Co nominal thickness of 5 and $10 \AA$. Wavelength of light: $632.8 \mathrm{~nm}$.

deposition of an AlN capping layer induces a transition from ferromagnetic state to superparamagnetic state. This transition could be due to the reduction of the "magnetic" size of the Co clusters or to the "magnetic disconnection" of the Co clusters. In both cases the transition can be understood with the formation of a dead magnetic layer at the interface between the AlN capping layer and the Co clusters similarly to the case of Fe layers capped with TiN (Ref. 32) where it has been shown that the nitrogen incorporation into the Fe lattice induces a reduction of the saturation magnetization. This dead magnetic layer results from the interaction of the AlN and the outermost Co atoms of the nanoclusters. As a consequence, the size of the "magnetic core" of the Co clusters is reduced and the effective magnetic size of the clusters reaches a value that is below the critical value that separates the ferromagnetic state from the super-paramagnetic state. Like in Fig. 8(a), the same phenomenon is observed for slightly bigger clusters (mean diameter $23 \mathrm{~nm}$ ) in Fig. 8(b) where it can be seen that the hysteresis loop of the AlN embedded Co clusters corresponds to Co clusters with a reduced amount of magnetic Co compared to the uncapped 


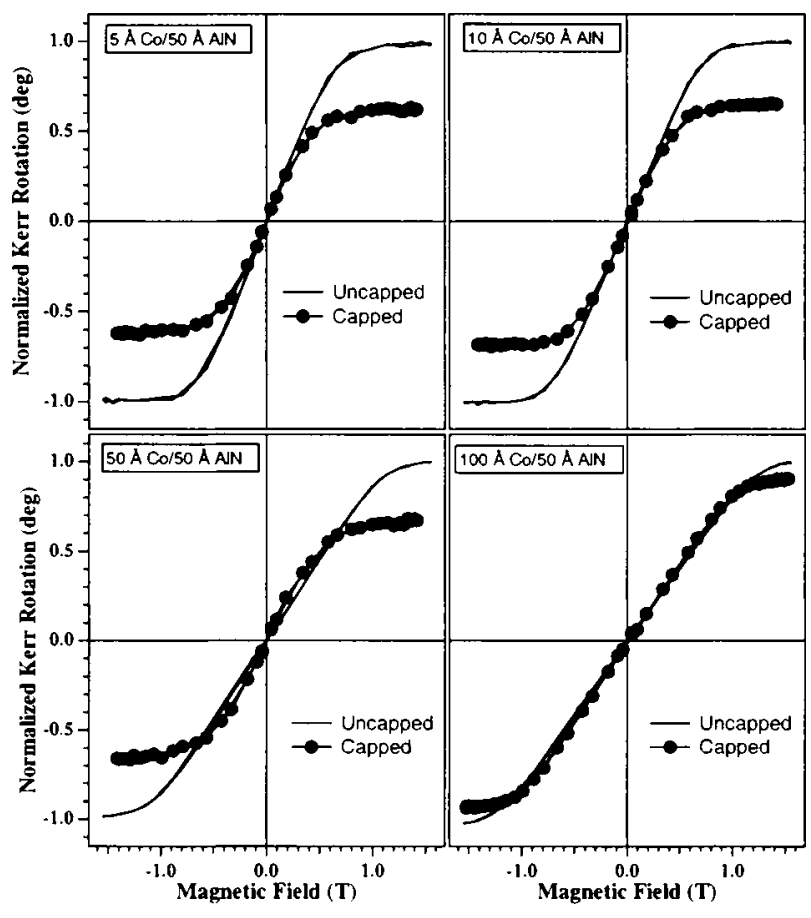

FIG. 9. Hysteresis loops measured in the polar Kerr configuration for AlN capped and uncapped Co/50 $\mathrm{AlN} /$ sapphire samples with Co nominal thickness of 5,10,50, and $100 \AA$. Wavelength of light: $650 \mathrm{~nm}$.

clusters. However, in that latter case the system is still ferromagnetic because of the limited thickness of the magnetic dead layer that does not allow the reduction of the magnetic size below the critical size. In order to determine the nature of this magnetic dead layer we have analyzed the evolution of the transversal Kerr response of a thin cobalt layer $(25 \AA)$ before and after the exposure to a nitrogen plasma and to the deposition of an $\mathrm{Al}$ capping layer. The hysteresis loops were measured in situ and the results showed that the hysteresis loops of the cobalt layers disappeared after the exposure to the nitrogen plasma, evidencing a strong reaction between the cobalt layer and the nitrogen plasma. On the contrary the deposition of a thin Al layer did not destroy the magnetic response; these results strongly suggest the formation of a nonmagnetic cobalt-nitride compound at the AlN/Co interface rather than an AlCo compound. The fact that no significant changes could be observed by comparing $\mathrm{x}$-ray results for the AlN capped and uncapped Co clusters is also consistent with the formation of a thin dead magnetic layer resulting from the interaction of the AlN and the outermost Co atoms of the nanoclusters and that is too thin to be resolved by XRD. We have further investigated this effect by measuring the Kerr hysteresis loops in the polar configuration and for different Co nominal thickness. The resulting hysteresis loops are displayed in Fig. 9. It can be observed that, like in transverse geometry, the polar Kerr loops show that the AlN capping layer has a strong effect on the magnetic properties of the Co clusters. The Kerr loops of Fig. 9 have been normalized in such way that the maximum Kerr rotation for uncapped Co nanoclusters is equal to 1 . It appears that the embedded clusters have always a lower Kerr rotation at saturation field and that the saturation field is reduced after deposition of the capping layer. Both effects are compatible with

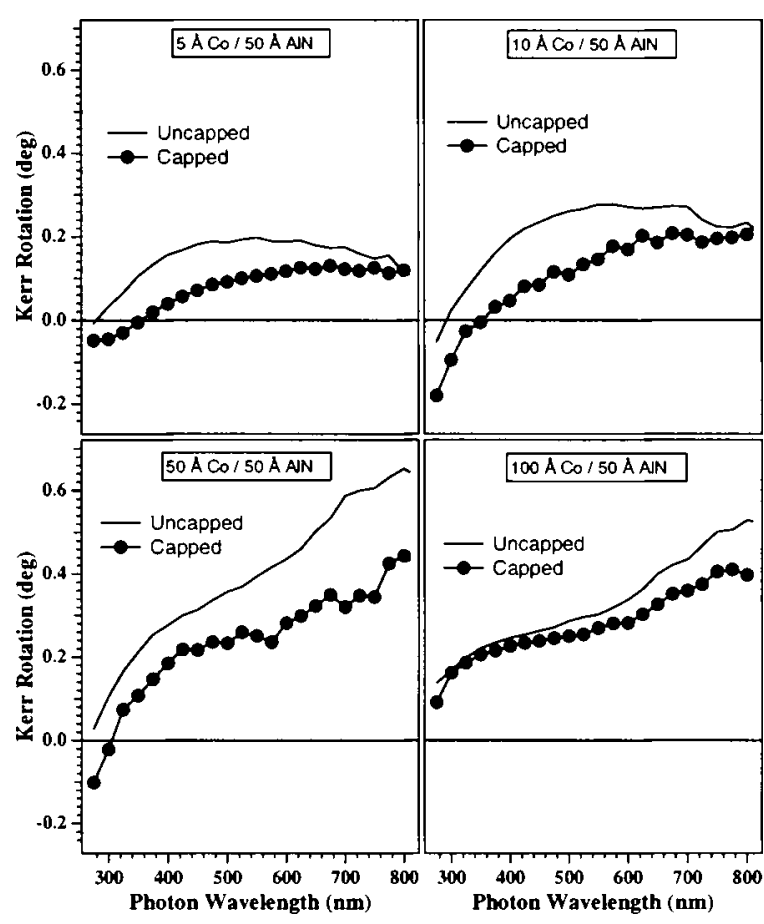

FIG. 10. Polar Kerr spectra measured at saturation field and for capped and uncapped samples of different Co nominal thickness.

the reduction of the magnetic size of the clusters in the direction perpendicular to the substrate plane, i.e., along the height of the Co clusters. Furthermore, it can be observed that such modifications of the magnetic properties are stronger for small Co particles indicating that the effect is localized at the interface between the Co clusters and the AlN capping layer (and probably at the AlN buffer substrate layer). Finally we have measured the polar Kerr spectra of a set of samples in order to investigate the photon energy dependence of the effect of the AlN matrix. The spectra are displayed in Fig. 10. Once more, the strong effect of the capping layer on the magneto-optical response of the Co nanoparticles is evidenced but in a wider photon wavelength range. It can be observed that, in the investigated photon energy range, the capping layer reduces significantly the Kerr rotation; also the effect is clearly more pronounced for the smallest Co nanoparticles which gives a confirmation that the observed effect is an interface effect and localized at the interface between the cobalt and the embedding AlN matrix. While the reduction of the Kerr rotation due to the AlN matrix is less pronounced for bigger clusters as a consequence of the interface effect, it can be observed that the reduction is more important at low wavelengths for small clusters and more important at higher wavelength for bigger clusters. Interestingly, such effect is also found in $\mathrm{Ni}$ and $\mathrm{Co}$ clusters embedded in AlN prepared either by ion implantation or by sputtering ${ }^{24,25}$ using for the deposition of AlN a sintered AlN target instead of growing the AlN layer by reactive sputtering as done here, or in Ni clusters embedded in a silicon nitride matrix. ${ }^{33}$ In those works an interaction between the matrix and the surface atoms of the clusters is suggested which produces a magnetically dead layer surrounding the clusters. This is likely to indicate that there is a general trend of nitride compound matrix to create a mag- 
netic dead layer when in contact to ferromagnetic transition metals.

\section{CONCLUSIONS}

We have presented a study on the properties of Co nanoclusters deposited on AIN and embedded in an AIN matrix. The growth conditions of the Co on AlN have been found to have a strong impact on the resulting system; apart from the growth mode that is known to depend on the deposition temperature, the size of the Co particles and their magnetic and magneto-optical properties have been found to depend strongly on the growth conditions. Also the interaction between the Co particles and the AIN has been directly evidenced by the study of Co particles embedded in an AlN matrix. In particular we have shown that the Kerr rotation is strongly reduced when the Co nanoclusters are embedded in AlN as well as the saturation magnetization and saturation field. Our results are compatible with a strong interaction between the Co and the adjacent AlN substrate and/or matrix. The interaction between the magnetic clusters and the matrices induces the formation of a dead magnetic layer at the interface between the Co clusters and the AlN matrix. Our results are likely to suggest that there is a general trend of nitride compound matrix to create a dead magnetic layer when in contact with ferromagnetic transition metals.

\section{ACKNOWLEDGMENTS}

This work was partially financed by the Spanish Commission of Science and Technology [Comisión Interministerial para la Ciencia Y la Tecnología (CICYT)], the Spanish Ministerio de Ciencia y Tecnología, and the Comunidad de Madrid (Project No. 07N/0108/2002). Y.H. acknowledges the Consejo Superior de Investigaciones Científicas (CSIC) and Ramón y Cajal program for financial support.

${ }^{1}$ I. M. L. Billas, A. Châtelain, and W. A. De Heer, Science 265, 1682 (1994).

${ }^{2}$ W. Chen and H. J. Ahmed, J. Vac. Sci. Technol. B 15, 1402 (1997).

${ }^{3}$ R. W. Cross, S. E. Russek, S. C. Sanders, M. R. Parker, J. A. Barnard, and S. A. Hossain, IEEE Trans. Magn. 30, 3825 (1995).

${ }^{4}$ S. E. Russek, R. W. Cross, S. C. Sanders, and J. O. Oti, IEEE Trans. Magn. 31, 3839 (1995).

${ }^{5}$ T. L. Hylton, M. A. Parker, K. R. Coffey, J. K. Howard, R. Fontana, and C. Tsang, Appl. Phys. Lett. 67, 1154 (1995).

${ }^{6}$ J. P. Bucher, D. C. Douglass, and L. A. Bloomfield, Phys. Rev. Lett. 66, 3052 (1991).
${ }^{7}$ S. E. Apsel, J. W. Emmert, J. Deng, and L. A. Bloomfield, Phys. Rev. Lett. 76, 1441 (1996).

${ }^{8}$ D. A. Eastham and I. W. Kirkman, J. Phys.: Condens. Matter 12, L525 (2000).

${ }^{9}$ J. Guevara, A. M. Llois, F. Aguilera-Granja, and J. M. MontejanoCarrizales, Solid State Commun. 111, 335 (1999), and references therein.

${ }^{10}$ J. L. Rodríguez-López, F. Aguilera-Granja, K. Michaelian, and A. Vega, Phys. Rev. B 67, 174413 (2003).

${ }^{11}$ Shangjr Gwo, Chung-Pin Chou, Chung-Lin Wu, Yi-Jen Ye, Shu-Ju Tsai, Wen-Chin Lin, and Minn-Tsong Lin, Phys. Rev. Lett. 90, 185506 (2003).

${ }^{12}$ H. A. Dür, S. S. Dhesi, E. Dudzik, D. Knabben, G. van der Laan, J. B. Goedkoop, and F. U. Hillebrecht, Phys. Rev. B 59 R701 (1999).

${ }^{13}$ R. Félix-Medina, J. Dorantes-Dávila, and G. M. Pastor, Phys. Rev. B 67, 094430 (2003).

${ }^{14}$ A. B. Klautau and S. Frota-Pessôa, Surf. Sci. 497, 385 (2002).

${ }^{15}$ J. C. Cezar, H. C. N. Tolentino, and M. Knobel, Phys. Rev. B 68, 054404 (2003).

${ }^{16}$ D. A. Eastham, P. M. Denby, A. Harrison, I. W. Kirkman, and A. G. Whittaker, J. Phys.: Condens. Matter 14, 605 (2002).

${ }^{17}$ J. Guevara, A. M. Llois, and M. Weissmann, Phys. Rev. Lett. 81, 5306 (1998).

${ }^{18}$ Y. Qiang, R. F. Sabiryanov, S. S. Jaswal, Y. Liu, H. Haberland, and D. J. Sellmyer, Phys. Rev. B 66, 064404 (2002).

${ }^{19}$ R. D. Sánchez, M. A. López-Quintela, J. Rivas, A. González-Penedo, A. J. García-Bastida, C. A. Ramos, R. D. Zysler, and S. Ribeiro Guevara, J. Phys.: Condens. Matter 11, 5643 (1999).

${ }^{20}$ M. Jamet, V. Dupuis, P. Mélinon, G. Guiraud, A. Pérez, W. Wernsdorfer, A. Traverse, and B. Baguenard, Phys. Rev. B 62, 493 (2000).

${ }^{21}$ M. Jamet, W. Wernsdorfer, C. Thirion, V. Dupuis, P. Mélinon, A. Pérez, and D. Mailly, Phys. Rev. B 69, 024401 (2004).

${ }^{22}$ J. M. Torres, F. Luis, L. M. García, J. Bartolomé, J. Stankiewicz, F. Petroff, F. Fettar, and A. Vaurés, J. Magn. Magn. Mater. 242-245, 575 (2002).

${ }^{23}$ J.-Y. Kim et al., Phys. Rev. Lett. 90, 017401 (2003).

${ }^{24}$ D. Zanghi, A. Traverse, F. Petroff, J.-L. Maurice, A. Vaurès, and J. P. Dallas, J. Appl. Phys. 89, 6329 (2001).

${ }^{25}$ D. Zanghi, C. M. Teodorescu, F. Petroff, H. Fischer, C. Bellouard, C. Clerc, C. Pélissier, and A. Traverse, J. Appl. Phys. 90, 6367 (2001).

${ }^{26} \mathrm{M}$. Jamet, V. Dupuis, C. Thirion, W. Wernsdorfer, P. Mélinon, and A. Pérez, Scr. Mater. 44, 1371 (2001).

${ }^{27}$ J. L. Menéndez, G. Armelles, A. Cebollada, C. Quintana, D. Ravelosona, C. Chappert, F. Peiró, and A. Cornet, Appl. Phys. Lett. 81, 1603 (2002).

${ }^{28}$ Y. Huttel, H. Gomez, A. Cebollada, G. Armelles, and M. I. Alonso, J. Cryst. Growth 242, 116 (2002).

${ }^{29}$ J. L. Menéndez, G. Armelles, C. Quintana, and A. Cebollada, Surf. Sci. 482-485, 1135 (2001); C. Martínez Boubeta, E. Navarroa, A. Cebollada, F. Briones, F. Peiró, and A. Cornet, J. Cryst. Growth 226, 223 (2001).

${ }^{30}$ F. Radu, M. Etzkorn, R. Siebrecht, T. Schmitte, K. Westerholt, and H. Zabel, Phys. Rev. B 67, 134409 (2003), and references therein.

${ }^{31}$ J. van Lierop, H. S. Isaacs, D. H. Ryan, A. Beath, and E. McCalla, Phys. Rev. B 67, 134430 (2003), and references therein.

${ }^{32}$ J. L. Menéndez et al., J. Appl. Phys. 89, 6314 (2001).

${ }^{33}$ M. Vila, F. Jiménez-Villacorta, C. Prieto, and A. Traverse, Phys. Scr., (in press); Mercedes Vila Juárez, Thesis, Universidad Autónoma de Madrid, 2003. 